\title{
Understanding CMP stacking hyperbola in terms of partial derivative wavefield
}

\author{
Changsoo Shin* and Seunghwan Chung $\ddagger$
}

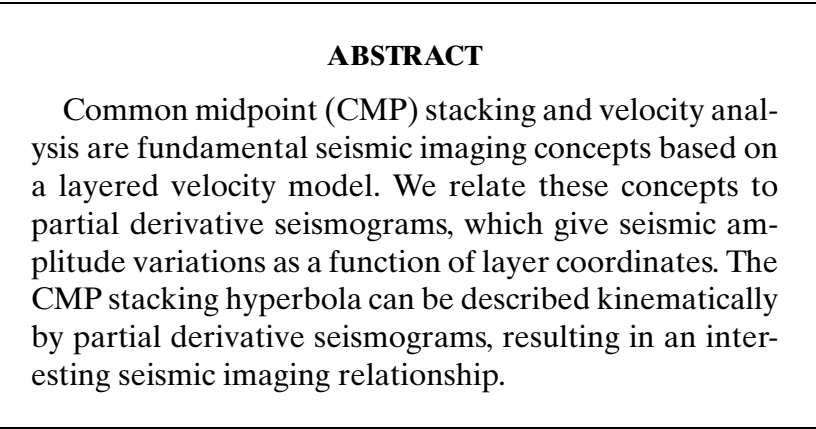

\section{INTRODUCTION}

In the years since Mayne (1962) invented the CDP technique as a fundamental data processing method, the ability of exploration seismologists to image the subsurface has been improved greatly. The main objective of summing seismic signals along a straight line after NMO correction is to enhance the primary reflected seismic signal and to suppress multiple reflections and noise. In stacking seismic data recorded and sorted in common midpoint (CMP) geometry, a hyperbolic time distance relation is assumed. This is a simple flat-layer model. However, this assumption fails for large source-receiver separations and more complex subsurface geology. An approach based on a direct model-based stacking method for the construction of an accurate zero-offset time image was proposed by Landa et al. (1993). The hyperbola having maximum coherence in velocity analysis is replaced by the traveltime curve of the CMP source-receiver configuration computed by ray tracing. Landa et al. (1993) summed the seismic signals along the traveltime curves computed by ray tracing and obtained an accurate zero offset section. In principle, there is no conceptual difference between the summation of seismic signals along the hyperbola and the summation of seismic signals along a straight line after NMO or dip moveout (DMO) correction. By comparing conventional CMP stacking and model-based stacking by Landa et al. (1993), we introduce the concept of a partial derivative seismogram with respect to the interface segment. The stacking hyperbola having maximum coherence in velocity analysis and the traveltime curve of Landa et al. (1993) can be evaluated as a first-arrival approximation to our partial derivative seismogram. In this paper, we show that the stacking hyperbola having the maximum coherence measurement in velocity analysis is, in fact, the kinematic approximation of the first-arrival events of the CMP partial derivative seismogram with respect to the reflection interface segment.

\section{PARTIAL DERIVATIVE SEISMOGRAMS WITH RESPECT TO THE INTERFACE PARAMETER}

As shown by Kelly et al. (1982), the 2-D scalar (i.e., acoustic) wave equation is given by

$$
\frac{\partial}{\partial x}\left(\frac{\partial U}{\rho \partial x}\right)+\frac{\partial}{\partial z}\left(\frac{\partial U}{\rho \partial z}\right)=\frac{\partial^{2} U}{k \partial t^{2}}+f(x, z, t),
$$

where $x$ is the horizontal distance, $z$ is the depth from the surface, $t$ is the time, $U(x, z, t)$ is the wavefield (i.e., displacement, pressure, etc.), $\rho(x, z)$ is the density, $k(x, z)$ is the bulk modulus, and $f(x, z, t)$ is the source function. This equation and the comparable elastic wave equation were solved by Marfurt (1984) using a finite-element approach. Equation (1) is based on Newton's second law of motion and Hooke's law, which, in the finite-element discretized formulation of the dynamic problem for linearly elastic, undamped materials, can be written as

$$
\mathbf{M u ̈}+\mathbf{K} U=\mathbf{f},
$$

where $\mathbf{M}$ is the mass matrix representing system inertia, $U$ is the wavefield vector, $\mathbf{K}$ is the stiffness matrix representing the Laplacian term in equation (1), and $\mathbf{f}$ is the source vector. The double dots indicate the second derivative with respect to time.

In finite-element discretization, the subsurface of the earth is subdivided into a continuous grid of triangular or quadrilateral elements. A wave equation with appropriate boundary conditions is approximated across each element. The solution

Manuscript received by the Editor September 3, 1997; revised manuscript received January 22, 1999.

${ }^{*}$ School of Civil, Urban \& Geosystem Engineering, Seoul National University, San 56-1, Sinlim-dong, Kwanak-ku, Seoul, 151-742, Korea. E-mail: css@model.snu.ac.kr.

$\ddagger$ Korea Institute of Geology, Mining and Materials, Gajung-Dong 30, Yousung-Gu, Taejon, Korea. E-mail: bigchung@rock25t.kigam.re.kr.

(C) 1999 Society of Exploration Geophysicists. All rights reserved. 
to the entire problem is then obtained by assembling the system of equations from each element and solving the resulting system of equations. Because of the increased computational effort required to represent an arbitrarily irregular grid, the finite-element discretization of the wave equations has found limited use in seismic modeling. Instead, the simpler, and therefore computationally faster, finite-difference method is more commonly used. However, when modeling wave propagation at an irregular interface, the finite-element method is superior to the finite-difference method. The finite-element technique is a powerful method for parameterizing the interfaces between differing geologic media. (A big advantage of the finiteelement method is that it can accurately represent slopes of interfaces, whereas finite-difference methods must use stairstep approximations.) The solution of any differential equation and the resulting mass and stiffness matrices by the finite-element method is a function of discretized coordinates, in addition to material parameters such as velocity and density.

The motivation for computing partial derivative seismograms with respect to the interface segment comes from needing a Jacobian matrix (sensitivity matrix) to solve the nonlinear least-squares inversion problem by moving the interface separating geologic media, as described by Shin (1988). To illustrate Shin's method, we apply equation (2) to an arbitrary model to compute the partial derivative wavefield with respect to the interface segment or the discretized interface coordinates. Figure 1 shows the 2-D model with two layers. The interface separating the layers has horizontal, oblique, and vertical segments. The interface points $\left(x_{m}, z_{m}\right)$ are shown for each segment. These three segments illustrate all possibilities. Suppose we slightly change the shape of the finite-length interface segment vertically by fixing both its end points in the horizontal layer (perturb the interface vertically) and then measure the difference between the pre- and postperturbed wavefields. This procedure results in an approximated measurement of the partial derivative wavefield with respect to the vertical coordinate of the interface. In perturbing the interface segment, velocity and density parameters are changed simultaneously.

In contrast to material parameter (velocity or density) perturbation used by Bleistein and Gray (1985), the amount of perturbation of velocity and density is the difference of both

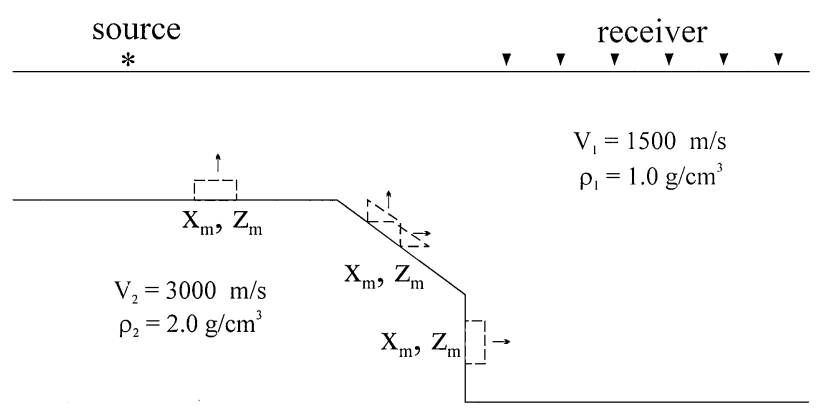

FIG. 1. The interface segments to be perturbed. The dotted line represents the interface shape when interface segment $z_{m}$ is perturbed. When perturbing the interface segment, velocity and density are perturbed as well. Unlike Born perturbation, the amount of perturbation is the difference between velocities sharing the interface. For the horizontal interface and the vertical interface, the interfaces can be perturbed in the vertical direction and the horizontal direction, respectively. For an interface with a dip, perturbation is done in the horizontal and vertical directions. the velocity and the density between geological media separated by the interface. This double perturbation is the major difference between material parameter perturbation and interface perturbation. Considering only material perturbation in the vicinity of the interface and by forcing the material parameter to be perturbed in a specific direction as if it were a vector, the results of material parameter perturbation are similar to those of our partial derivative seismogram. In other words, this procedure is equivalent to taking the partial derivative of the wavefield with respect to the vertical coordinate or differentiating equation (2) with respect to $z_{m}$. The result of this differentiation gives

$$
\mathbf{M} \frac{\partial \ddot{\mathbf{u}}}{\partial z_{m}}+\mathbf{K} \frac{\partial \mathbf{u}}{\partial z_{m}}=-\frac{\partial \mathbf{M}}{\partial z_{m}} \ddot{\mathbf{u}}-\frac{\partial \mathbf{K}}{\partial z_{m}} \mathbf{u}=\mathbf{f}^{*} .
$$

Differentiation with respect to $x_{m}$ and $z_{m}$ for oblique segments and with respect to $x_{m}$ for vertical segments results in an equation similar to equation (3).

Equation (3) is in the same form as equation (2), where $\ddot{\mathbf{u}}$, $\mathbf{u}$, and $\mathbf{f}$ are replaced by $\partial \ddot{\mathbf{u}} / \partial z_{m}, \partial \mathbf{u} / \partial z_{m}$, and $\mathbf{f}^{*}$, respectively. Since $\mathbf{f}$ in equation (2) is not a function of the interface coordinate unless we excite the source at the interface, the derivative of $\mathbf{f}$ in equation (2) with respect to the interface segment coordinate becomes zero. The value $\mathbf{f}^{*}$ is a virtual source used to compute the partial derivative wavefield $\partial \mathbf{u} / \partial z_{m}$. The virtual source is an imaginary source required to compute the partial derivative wavefield. Using the virtual source as the real source, we can solve equation (3) for partial derivative wave propagation (i.e., partial derivative seismograms). Physically, a partial derivative seismogram represents the amount of energy that travels through the interface after being radiated from the source. By looking at the partial derivative seismogram, we can tell which part of the wavefield measured at the surface passes through each interface segment in the subsurface. By measuring the zero-lag value of crosscorrelation between the field seismogram and the partial derivative seismogram, we can tell how much of the field seismogram is sensitive to the interface segment. We can then image the subsurface by displaying the zerolag crosscorrelation between the field seismogram and the partial derivative seismogram at the perturbed interface position.

Another way of viewing this is found in the least-squares inversion and migration started by Tarantola (1984). Gray (1997) discusses the relation between least-sqaures inversion and migration by comparing the seismic inversion and migration developed by three leading scientists. Least-squares inversion degenerates into migration by ignoring the off-diagonal terms of the approximate Hessian (Gray, 1997; Pratt et al., 1998) or by adding a large constant to the main diagonal of the approximate Hessian matrix as part of the regularization process (Lines, 1998, personal communication).

We now explain perturbation for an oblique (i.e., dipping) layer and its implication on the CMP stacking theory. Figure 2 shows that flat layers, exemplified by the top layer, act as singlepoint reflectors. In comparison, dipping layers do not act as single-point reflectors but have finite-sized smear segments of reflected rays. Despite the fact that the smearing effect profoundly alters reflections compared to point reflections, the (DMO) theory resolves smearing from dipping layers. The rays for a CMP gather in the flat layered media are focused exactly on the CDP; however, the rays for the CMP gather from the dipping layer are unfocused because of smearing. To explain the 
theoretical basis of CMP stacking to include smearing, we can use a partial derivative seismogram with respect to finite-length segments of the interface (i.e., the smearing zone). When perturbing the finite-size interface segment for an oblique layer, we replace a CMP gather seismogram with a CMP gather partial derivative seismogram generated by an oblique bar-shaped virtual source.

\section{CMP STACKING HYPERBOLA IN TERMS OF PARTIAL DERIVATIVE SEISMOGRAMS}

To illustrate the CMP stacking hyperbola in terms of partial derivative seismograms, we use a four-layer velocity model as shown in Figure 3. Within this model, we identify ten interface segments and number them 1 through 10 . Segments 2, 5, 8,

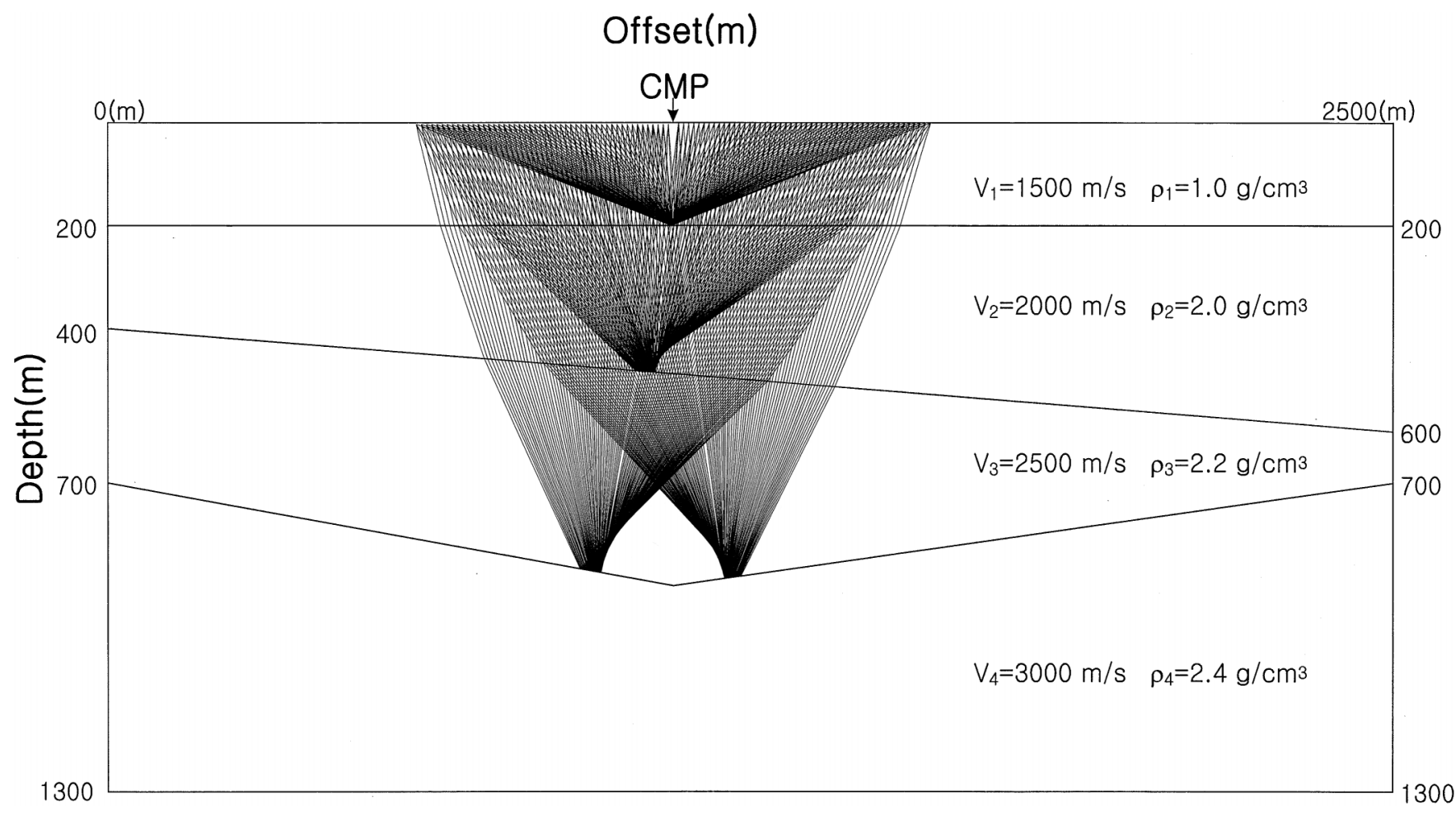

FIG. 2. Raypath diagrams for a CMP source-receiver configuration. Rays for the flat layer are focused on the CMP point exactly, whereas rays for the dipping layer and synclinal model are unfocused. When perturbing the interface segment for the CMP source-receiver configuration, the length of the perturbed zone can vary from a point for the flat layer to a relatively long segment for the dipping and other layers.

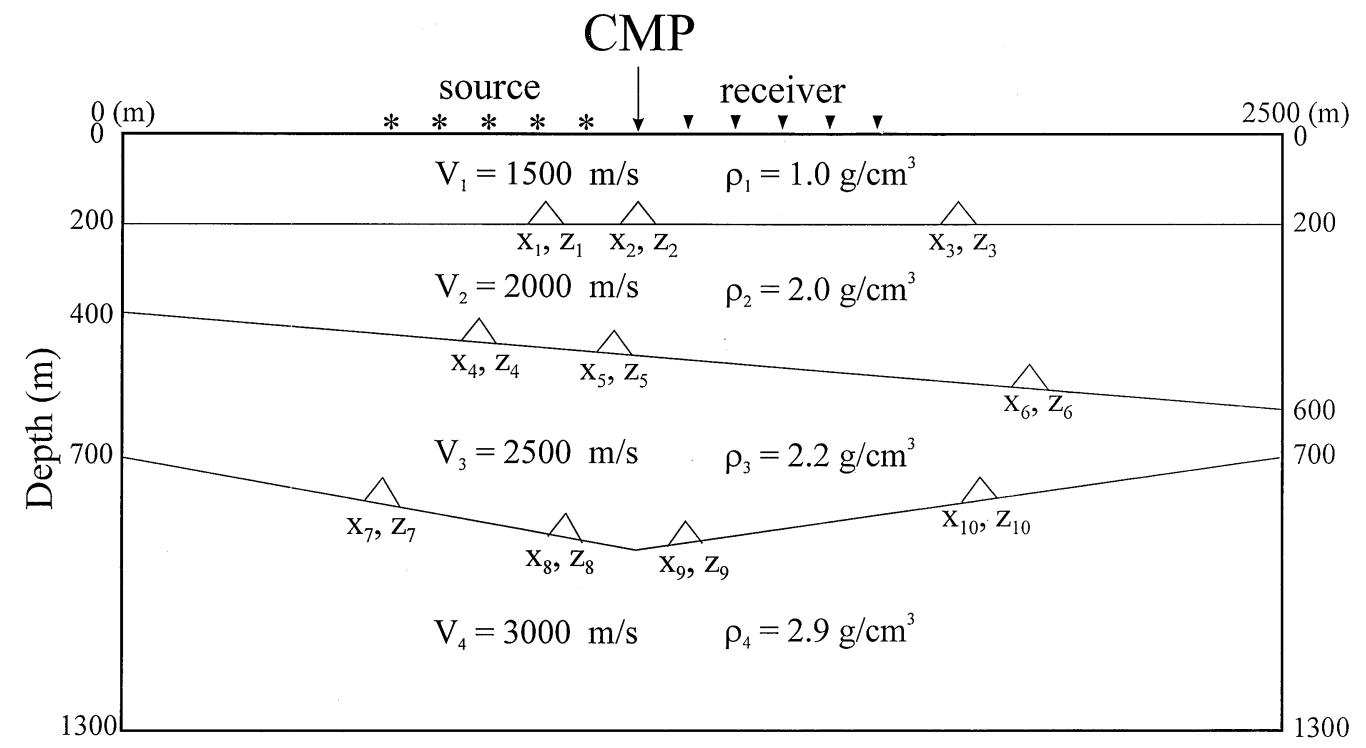

FIG. 3. Ten interface segments are perturbed vertically and horizontally. The CMP point is located at $1100 \mathrm{~m}$. Every sixth source and receiver are indicated by an asterisk and a triangle, respectively. Forty-five shot-receiver pairs are used to compute the forward modeling data and the partial derivative seismograms with respect to the interface segments. 
and 9 were specifically noted for later analysis; the remaining segments are arbitrary.

When we perturb an interface segment, we have an unlimited number of choices for perturbing the interface segment by shifting and adjusting the length of the interface segment. The length of the interface segment to be perturbed can vary from one point to a relatively long interface segment, depending upon the length of the focused or unfocused (smeared) CMP zone. In fact, we should calculate the unlimited number of CMP partial derivative seismograms with respect to the unlimited number of interface segments, but it is impossible for us to generate every possible CMP partial derivative seismogram with respect to an unlimited interface segment.

We perturbed all ten segments vertically and seven segments (4 through 10) horizontally. For the horizontal interface between the first and second layers, we used point perturbation. For the dipping layers, we used finite-segment perturbations with smear lengths ranging from 8 to 14 nodal points. Based on Figure 2, we calculated the smear zone lengths so we could perturb the same length of the interface segment with the finiteelement modeling technique. Because of its greater diversity for multiple-shot simulations, we transformed equation (2) into the frequency domain and used a frequency domain finiteelement technique to compute the CMP gather and partial derivative seismograms for a CMP at $1100 \mathrm{~m}$ (Figure 3). For simplicity, to avoid data processing steps and CMP sorting, and to save computation time, we calculated the CMP seismograms and CMP partial derivative seismograms for 45 shot-receiver pairs instead of computing several hundred multiple-shot seismograms.

Figure 4 shows the CMP gather seismograms, and Figure 5 shows the 17 partial derivative seismograms with respect to the interface segments for the 10 segments shown in Figure 3. It is obvious that for the first interface, the partial derivative seismogram with respect to $z_{2}$ (Figure $5 \mathrm{~b}$ ) fits the CMP gather in Figure 4 better than do the partial derivative seismograms

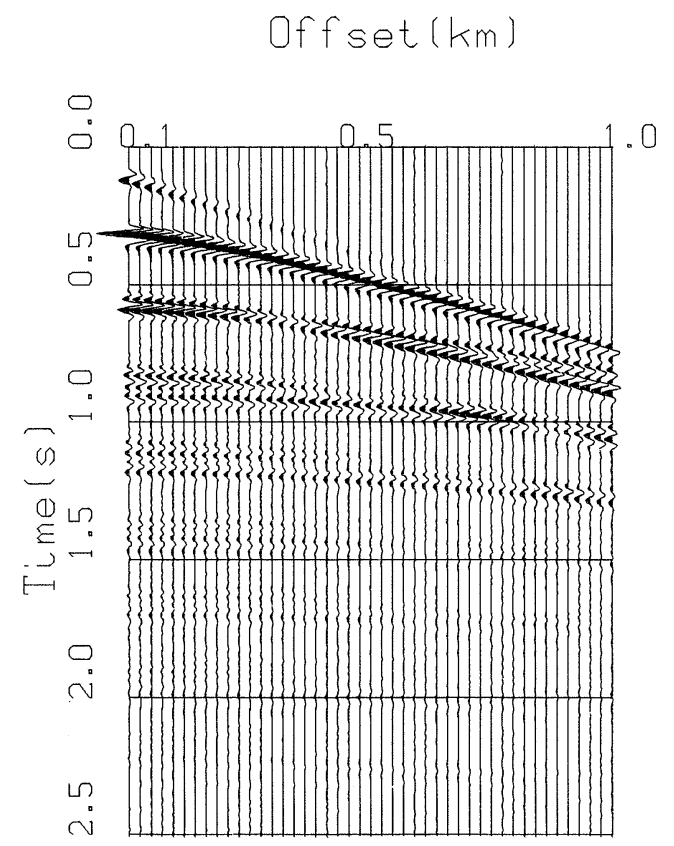

FIG. 4. CMP gather section of forward modeling data for a CMP located at $1100 \mathrm{~m}$. shown in Figures 5a,c. There is a good correlation between reflection events (primaries and multiples) from the first interface on the CMP gather and the partial derivative seismogram. For the second interface, the partial derivative seismograms with respect to interface segments $x_{5}$ and $z_{5}$ (Figures $5 \mathrm{f}$ and $5 \mathrm{~g}$ ) have maximum correlation with the CMP gather in Figure 4. For the third interface, the partial derivative seismograms in Figures 51-o, have maximum correlation with the CMP gather in Figure 4 . To clearly demonstrate the connection between the partial derivative seismogram and the CMP stacking hyperbola, we computed the velocity spectrum for Figure 4. Figure 6 shows the velocity spectrum. Figure 7 shows the stacking hyperbolas (the traveltime curves calculated using the approach of Landa et al., 1993) corresponding to the three reference times and the corresponding three rms velocities numbered in Figure 6 for the primary reflections in Figure 4. Hyperbola 1 in Figure 7 overlays the first-arrival event of the partial derivative seismogram from segment 2 (Figure 5b). Hyperbolas 2 and 3 overlay the first-arrival events in Figures 5f,g, and 5l,m,n,o, respectively. The first-arrival events of the partial derivative seismograms correspond to those primary reflections usually taken into account in conventional stacking.

To proceed further, we computed zero-lag values of crosscorrelation between the partial derivative seismogram and the CMP gather seismograms. In the same way that the partial derivative seismograms are defined with respect to the $x$ - and $z$-coordinates of the interface segment, vector zero-lag crosscorrelation with respect to the interface segment can be expressed as

$$
s_{x}=\int_{-x_{\max }}^{x_{\max }} \int_{0}^{t_{\max }} u(x, t) u_{x}(x, t) d x d t
$$

and

$$
s_{z}=\int_{-x_{\max }}^{x_{\max }} \int_{0}^{t_{\max }} u(x, t) u_{z}(x, t) d x d t,
$$

where $u(x, t)$ is the CMP gather seismogram, $u_{x}(x, t)$ is the CMP gather partial derivative seismogram with respect to the $x$-coordinate of the interface segment, $u_{z}(x, t)$ is the CMP partial derivative seismogram with respect to the $z$-coordinate of the interface segment, $x$ is the offset distance, $x_{\max }$ is the maximum offset distance, $t_{\max }$ is the maximum recording time, $s_{x}$ is the zero-lag value of crosscorrelation with respect to the $x$ coordinate of the interface segment, and $s_{z}$ is the zero-lag value crosscorrelation with respect to the $z$-coordinate of the interface segment. The total value of the zero-lag crosscorrelation can be expressed as a vector whose magnitude and direction are given by

$$
s=\sqrt{s_{x}^{2}+s_{z}^{2}}
$$

and

$$
\theta=\tan ^{-1} \frac{s_{z}}{s_{x}} .
$$

An interesting observation is that angle $\theta$ in equation (7) is approximately the same as the dip of the interface segment. Table 1 shows dipping angles computed by equation (7) for interface segments 2, 5, 8, and 9, as shown in Figure 3. Dips computed using equation (7) approximate dips of the interface 


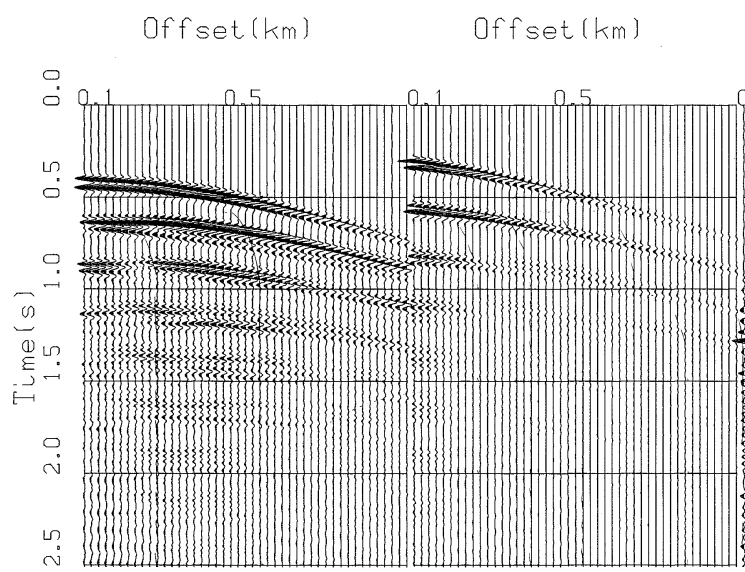

Offset $(\mathrm{km})$

Offset $(\mathrm{km})$

Offset $(\mathrm{km})$

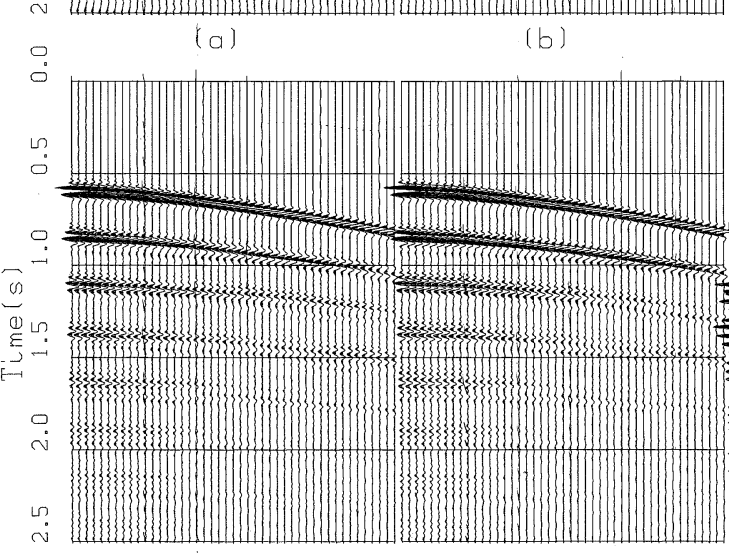

(b)

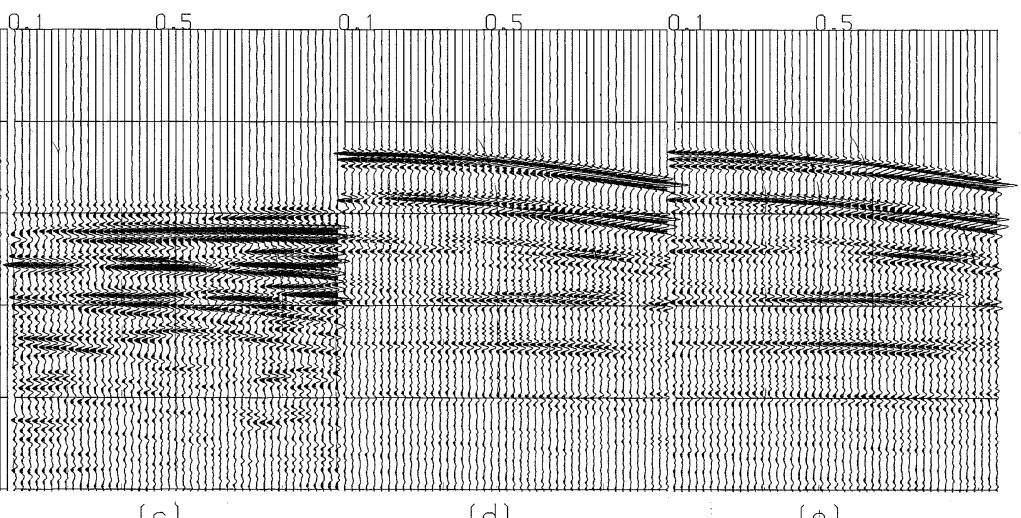

(c)

(d)

(e)

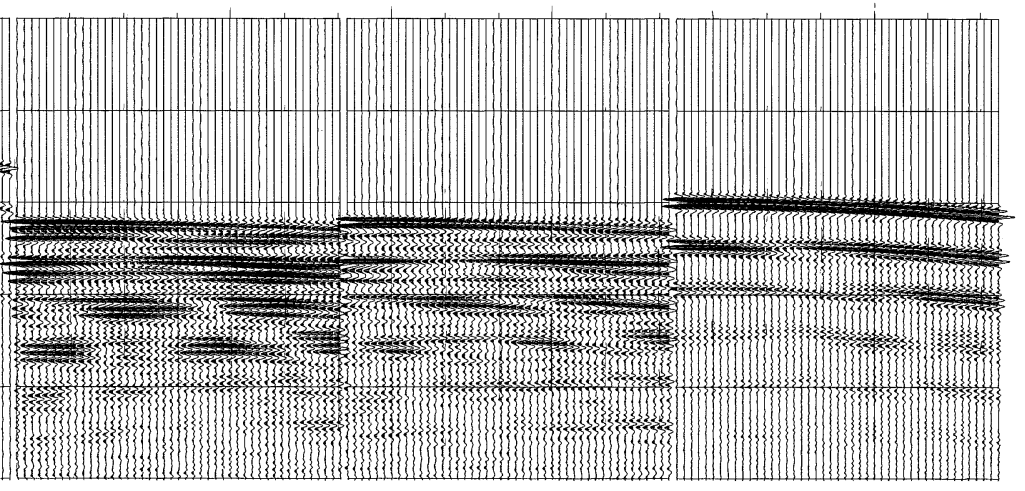

(f)

$(g)$

( h )

(i)

$(j)$
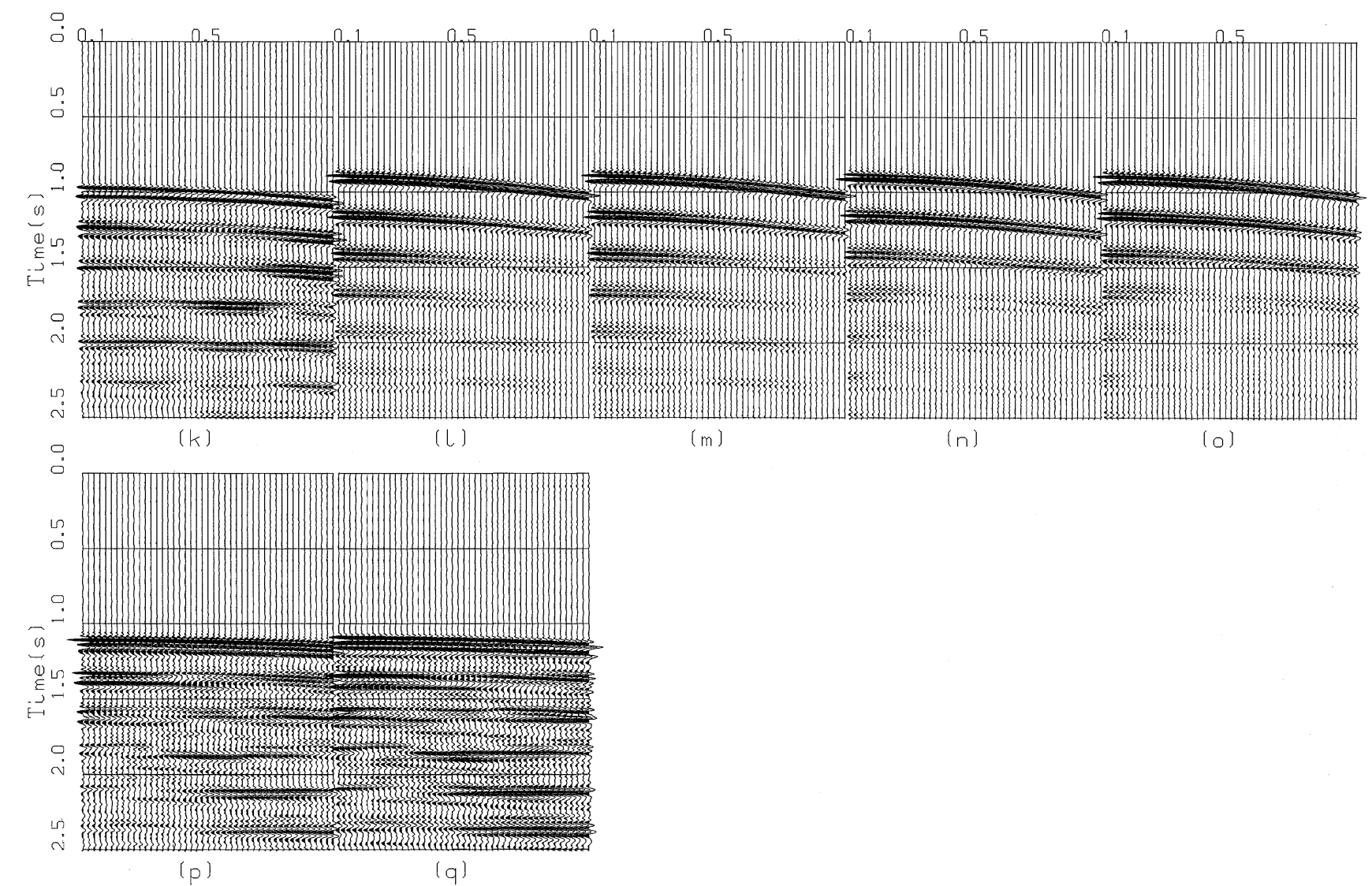

$(\mathrm{m})$

(n)

(o)

FIG. 5. CMP gather section of partial derivative seismograms with respect to the following interface segments in Figure 3: (a) $z_{1}$, (b) $z_{2}$, (c) $z_{3}$, (d) $x_{4}$, (e) $z_{4}$, (f) $x_{5}$, (g) $z_{5}$, (h) $x_{6}$, (i) $z_{6}$, (j) $x_{7}$, (k) $z_{7}$, (l) $x_{8}$, (m) $z_{8}$, (n) $x_{9}$, (o) $z_{9}$, (p) $x_{10}$, and (q) $z_{10}$. 


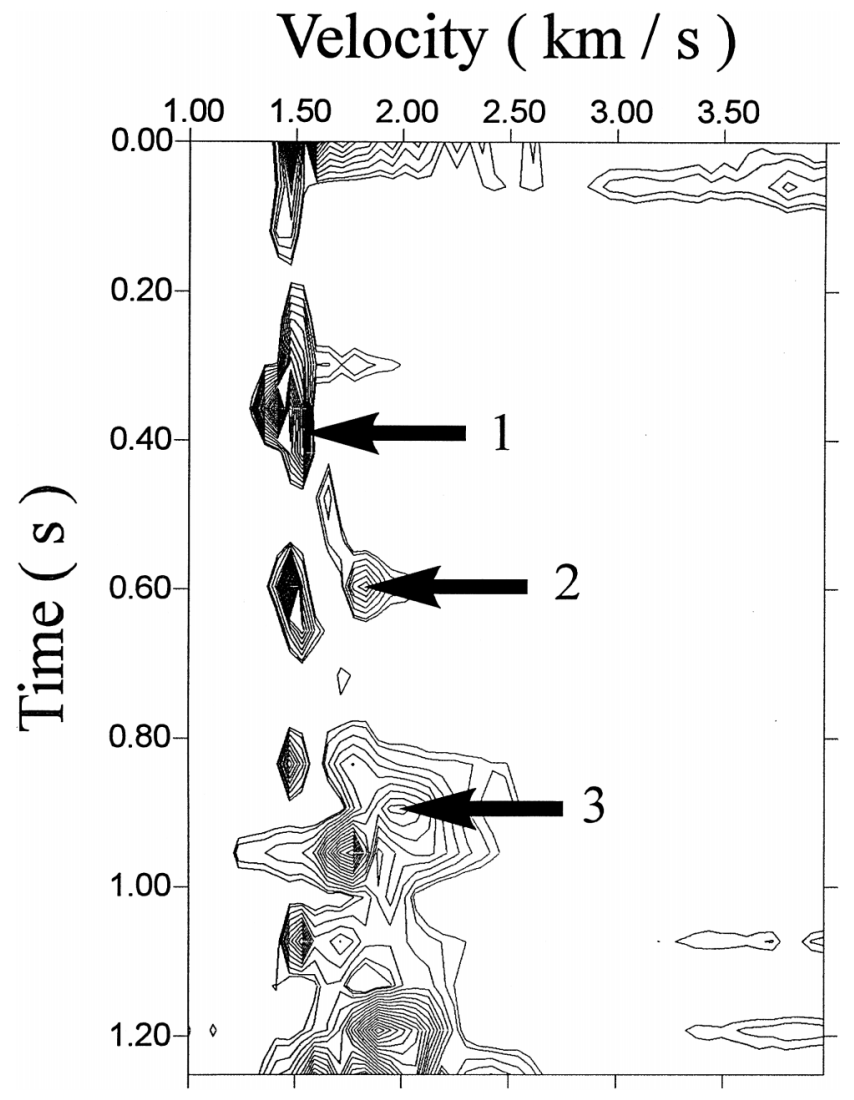

FIG. 6. Velocity spectrum for the section shown in Figure 4. Arrows 1,2, and 3 indicate the primary reflections from the flat layer, dipping layer, and synclinal layer, respectively, in Figure 2.

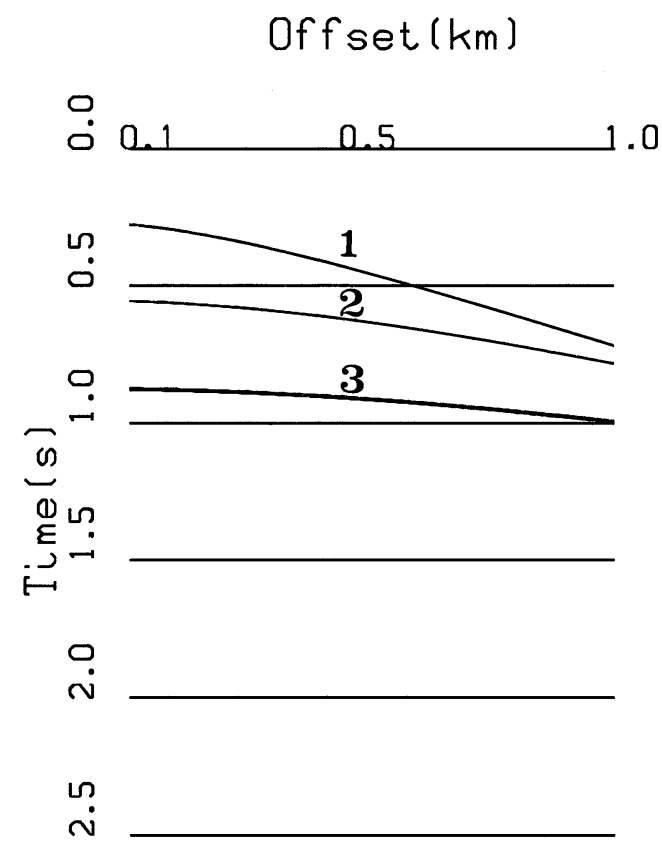

FIG. 7. Stacking hyperbolas reproduced from the velocity spectrum (the traveltime curves calculated using the approach of Landa et al., 1993). The first hyperbola matches the first-arrival part of Figure 5b. The second hyperbola matches the first-arrival parts of Figures $5 \mathrm{f}$ and $5 \mathrm{~g}$. The third hyperbola matches the first-arrival parts of Figures 51-5o. segments. However, in the usual stacking procedure, one measures the maximum coherence using a trial hyperbola that represent a kinematic waveform approximation of the first-arrival part of the partial derivative seismograms with respect to a certain interface segment. Since it is impossible to determine the exact partial derivative seismogram unless one knows the velocity model and computes the partial derivative seismogram using numerical modeling techniques, it is difficult to tell which partial derivative seismogram (with respect to the $x$ - or the $z$ coordinate of the interface segment) is kinematically approximated in conventional stacking. The mathematical expression of conventional CMP stacking can be given as

$$
s=\int_{-x_{\max }}^{x_{\max }} \int_{0}^{t_{\max }} u(x, t) \delta\left(t-\sqrt{t_{0}^{2}+\frac{x^{2}}{v_{s}^{2}}}\right) d x d t,
$$

where $\delta$ is a delta function and $v_{s}$ is the rms velocity. A $\delta$ function in equation (8) represents the kinematic waveform expression of the CMP partial derivative seismogram. In a distributional sense, equation (8) means summing seismic signals along the hyperbola. In conventional stacking, the correct position for displaying a zero-lag value of crosscorrelation is unknown. For flat layered media, the summed signal having maximum zerolag value of crosscorrelation occurs just below the CMP. The stacked signal is displayed at zero-offset time $t_{0}$, which is the shortest time and is proportional to the depth of the interface segment. Migration procedures put stacked signals into their correct positions.

To examine the link between stacking and prestack migration, we stacked the CMP gather seismogram shown in Figure 4. Figure 8 shows the stacked trace for a CMP gather seismogram

Table 1. Dips of the interface segments in Figure 3.

\begin{tabular}{ccc}
\hline Segment & True dip & Dip computed by equation (7) \\
\hline 2 & $0^{\circ}$ & $0^{\circ}$ \\
5 & $-4.57^{\circ}$ & $-4.39^{\circ}$ \\
8 & $-10.30^{\circ}$ & $-11.92^{\circ}$ \\
9 & $8.13^{\circ}$ & $9.3^{\circ}$ \\
\hline
\end{tabular}

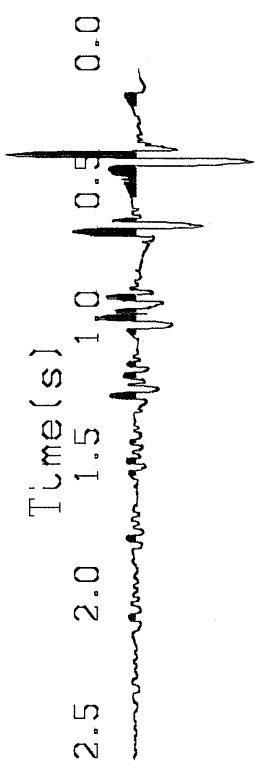

FIG. 8. Stacked trace for the CMP gather section of Figure 4. 
shown in Figure 4; Figure 9 shows the total zero-lag value of crosscorrelation displayed on the depth model. Figure 9 illustrates that a given stacked trace displayed below CMP can be moved to the correct depth positions by measuring the total zero-lag value of crosscorrelations and displaying them at their correct location. In the illustrated case, the total zero-lag value of crosscorrelation is the number indicating the interface separating the geologic layers (there is no interface like wiggle).

Another problem arises when measuring a zero-lag value of crosscorrelation. Geometric spreading and reflection coefficient changes in wave propagation through the geologic media cause a small zero-lag value of crosscorrelation at depth points remote from the source. In field seismograms, such small values of crosscorrelation are compensated by automatic gain control (AGC) of the seismograms. Our recourse is to normalize the total zero-lag value of crosscorrelation. We used three different ways of normalizing the zero-lag crosscorrelation: responding to the maximum coherence in velocity analysis and Landa et al.'s (1993) traveltime curve represent the kinematics of the first-arrival event of the CMP partial derivative seismogram with respect to the reflection interface segment. The high cost of computing partial derivative seismograms makes stacking which uses the partial derivative seismogram impractical unless we use a more efficient way (using source and receiver reciprocity) of computing partial derivative seismograms. Therefore, it is interesting to see the relationship of partial derivative seismograms to CMP stacking hyperbolas (which are inexpensive). If one computes the partial derivative seismogram using a numerical modeling technique-although the procedure is expensive-one can obtain the stacking hyperbola, including primaries, multiples, and other wave events. These hyperbolas will have amplitude and phase variations along the hyperbolic curves. As a result, if the partial derivative seismograms are used for subsurface imaging, we do not

$$
\begin{gathered}
s=\sqrt{\frac{\left(\int_{-x_{\max }}^{x_{\max }} \int_{0}^{t_{\max }} u(x, t) u_{x}(x, t) d x d t\right)^{2}+\left(\int_{-x_{\max }}^{x_{\max }} \int_{0}^{t_{\max }} u(x, t) u_{z}(x, t) d x d t\right)^{2}}{\left(\int_{-x_{\max }}^{x_{\max }} \int_{0}^{t_{\max }} u_{x}(x, t) u_{x}(x, t) d x d t\right)^{2}+\left(\int_{-x_{\max }}^{x_{\max }} \int_{0}^{t_{\max }} u_{z}(x, t) u_{z}(x, t) d x d t\right)^{2}},} \\
s=\frac{\left(\int_{-x_{\max }}^{x_{\max }} \int_{0}^{t_{\max }} u(x, t) u_{x}(x, t) d x d t\right)^{2}+\left(\int_{-x_{\max }}^{x_{\max }} \int_{0}^{t_{\max }} u(x, t) u_{z}(x, t) d x d t\right)^{2}}{\sqrt{\left(\int_{-x_{\max }}^{x_{\max }} \int_{0}^{t_{\max }} u_{x}(x, t) u_{x}(x, t) d x d t\right)^{2}+\left(\int_{-x_{\max }}^{x_{\max }} \int_{0}^{t_{\max }} u_{z}(x, t) u_{z}(x, t) d x d t\right)^{2}}},
\end{gathered}
$$

and

$$
s=\frac{\left(\int_{-x_{\max }}^{x_{\max }} \int_{0}^{t_{\max }} u(x, t) u_{x}(x, t) d x d t\right)^{2}+\left(\int_{-x_{\max }}^{x_{\max }} \int_{0}^{t_{\max }} u(x, t) u_{z}(x, t) d x d t\right)^{2}}{\left(\int_{-x_{\max }}^{x_{\max }} \int_{0}^{t_{\max }} u_{x}(x, t) u_{x}(x, t) d x d t\right)^{2}+\left(\int_{-x_{\max }}^{x_{\max }} \int_{0}^{t_{\max }} u_{z}(x, t) u_{z}(x, t) d x d t\right)^{2}},
$$

Figure 10 shows three different sections normalized by equation 9. From Figure 10, we note that the values are very small for all but four locations. After normalization, the deeper image of interfaces is enhanced so we can identify them more easily. Since we did not generate every possible partial derivative seismogram, it is difficult to determine which normalization gives the best enhanced image. However, these normalizations provide a logical way to obtain the subsurface image without using automatic gain recovery of seismic signals weakened by geometric spreading and reflection coefficient changes. These sections can be considered as prestack depth migrations of a CMP gather seismogram of Figure 4.

\section{CONCLUSION}

This paper proposes a new approach for a kinematic evaluation of the CMP stacking hyperbola by using the partial derivative seismograms needed for full waveform inversion. Numerical tests indicate that the CMP stacking hyperbola cor- need AGC of the seismic signals. The normalization process will compensate for the small zero-lag value of crosscorrelation caused by geometric spreading.

\section{ACKNOWLEDGMENT}

This paper was inspired by Dr. Kurt J. Marfurt of Amoco Production Company, Dr. Franklyn Levin of Western Geophysical, and Dr. Larry Lines of the University of Calgary while Shin worked at Amoco as a consultant. We are extremely grateful to Dr. Franklyn Levin for helping us rewrite this paper.

\section{REFERENCES}

Bleistein, N., and Gray, S. H., 1985, An extension of the Born inversion method to a depth dependent reference profile: Geophys. Prosp., 33, 999-1022.

Gray, S. H., 1997, True-amplitude seismic migration: A comparison of three approaches: Geophysics, 62, 929-936.

Kelly, K. R., Alford, R. M., and Whitmore, N. D., 1982, Modeling- 


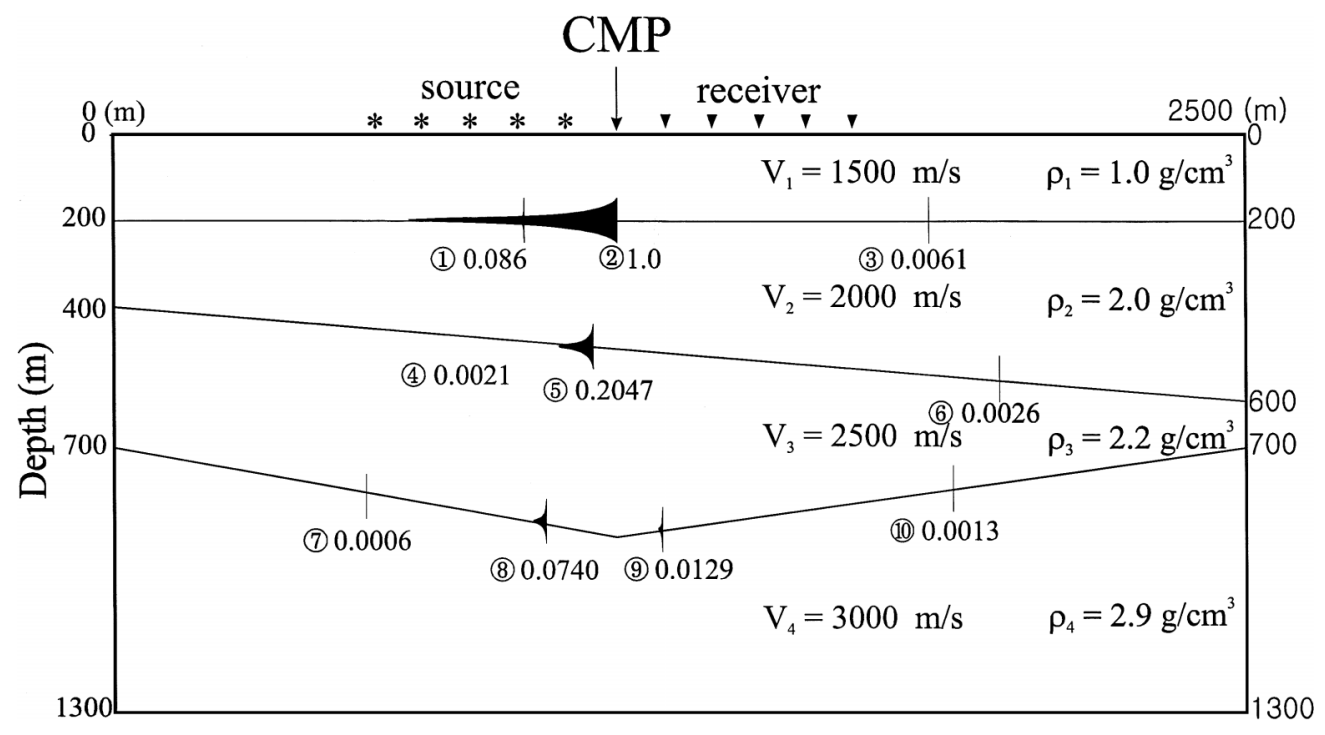

FIG. 9. Prestack depth-migrated section for the CMP gather section of Figure 4. The total zero-lag crosscorrelation is displayed at the interface locations shown in Figure 3. These values, except for four locations, are too small to be displayed.

The forward method, in Jain, K. C., and DeFigueiredo, R. P., Eds., Concepts and techniques in oil and gas exploration: Soc. Expl. Geophys., 91-114.

Landa, E., Thore, P., and Reshef, M., 1993, Model-based stack: A method for constructing an accurate zero-offset section for complex overburdens: Geophys. Prosp., 41, 661-670.

Marfurt, K. J., 1984, Accuracy of finite-difference and finite-element modeling of the scalar and elastic wave equations: Geophysics, 49, 533-549.
Mayne, W. H., 1962, Common reflection point horizontal stacking technique: Geophysics, 27, 927-928.

Pratt, R. G., Shin, C., and Hicks, J. G, 1998, Gauss-Newton and full Newton method in frequency-space seismic waveform inversion: Geophys. J. Internat., 133, 341-362.

Shin, C., 1988, Nonlinear elastic wave inversion by blocky parameterization: Ph.D thesis, Univ. of Tulsa.

Tarantola, A., 1984, Inversion of seismic reflection data in the acoustic approximation: Geophysics, 49, 1259-1266. 
a)

\section{CMP}

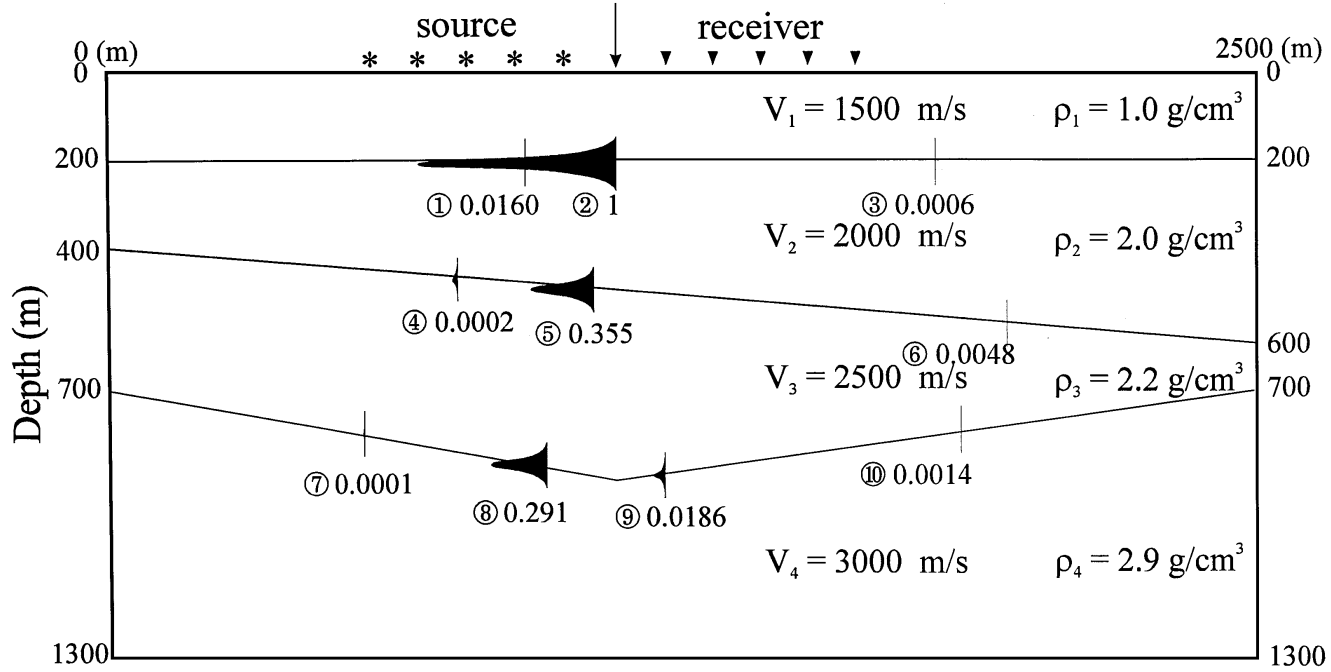

b)

\section{CMP}

source receiver

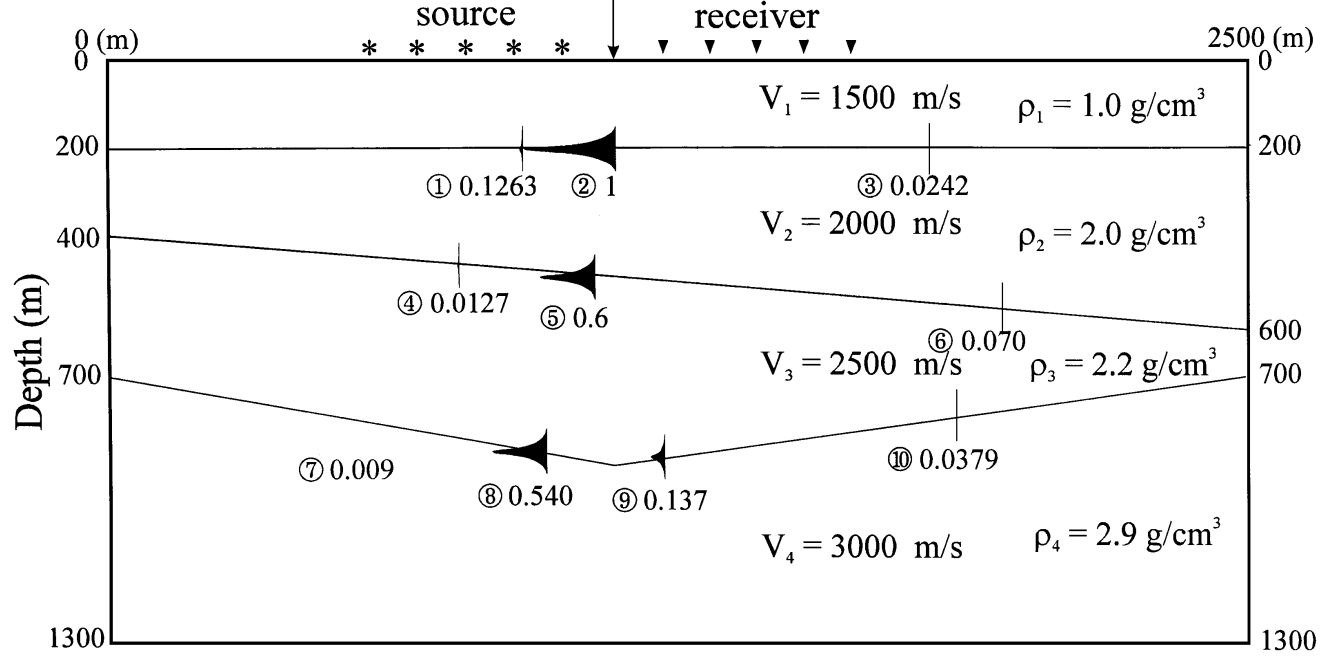

c)

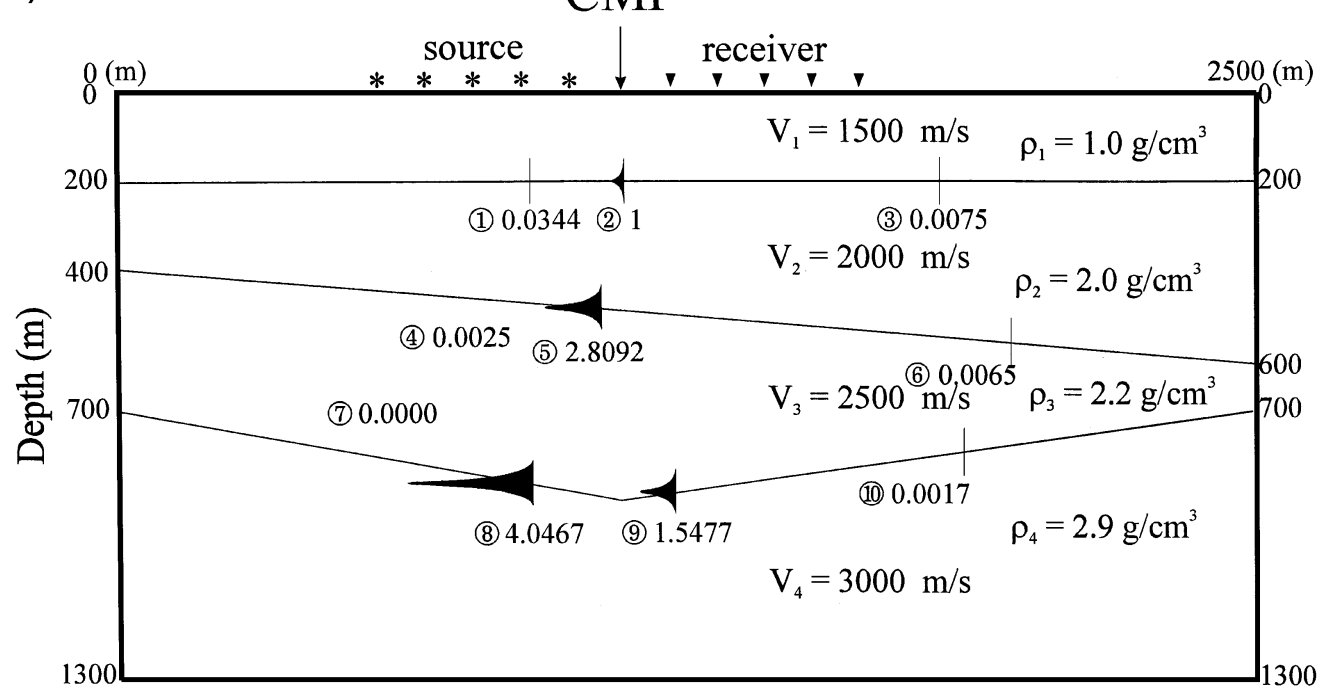

FIG. 10. Normalized total zero-lag values of crosscorrelation displayed for the interface locations shown in Figure 3 . The values are very small for all but four locations. The normalized total zero-lag value of crosscorrelation is displayed on each interface segment. (a) Depth section normalized by equation (9a). (b) Depth section normalized by equation (9b). (c) Depth section normalized by equation $(9 \mathrm{c})$. 